\title{
Phronesis
}

\section{Le stage en formation alternée dans l'enseignement supérieur : pour quel développement professionnel ?}

\section{Le cas d'une formation universitaire aux métiers de la} formation

\section{The internship training alternating in higher education: what kind of professional development?} The case of a university education for careers in training

\section{Jérôme Eneau, Geneviève Lameul et Éric Bertrand}

Volume 3, numéro 1-2, janvier-avril 2014

Le stage en formation, tendances et résistances

URI : https://id.erudit.org/iderudit/1024587ar

DOI : https://doi.org/10.7202/1024587ar

Aller au sommaire du numéro

Éditeur(s)

Université de Sherbrooke

ISSN

1925-4873 (numérique)

Découvrir la revue

Citer cet article

Eneau, J., Lameul, G. \& Bertrand, É. (2014). Le stage en formation alternée dans l'enseignement supérieur : pour quel développement professionnel ? Le cas d'une formation universitaire aux métiers de la formation. Phronesis, 3(1-2), 38-48. https://doi.org/10.7202/1024587ar
Résumé de l'article

Cette recherche part de l'analyse de dix documents réflexifs, produits en fin d'année dans le cadre de l'accompagnement à la professionnalisation par des étudiants de master 1 et master 2 SIFA (Stratégie et ingénierie en formation d'adultes) de l'Université Rennes 2 . Cette production réflexive, qui vise à formaliser et expliciter l'articulation des approches théoriques et praxéologiques caractéristique de ce Master, rend compte du vécu de stage en alternance à visée intégrative, de la part des étudiants. L’analyse de ces écrits permet de caractériser le processus de leur développement professionnel à partir d'un modèle visant à articuler trois dimensions : 1) les dynamiques identitaires en jeu dans ce processus de professionnalisation ; 2) la construction d'une socialisation professionnelle progressive ; 3) l'élaboration d'une posture spécifique de praticien-réflexif. La méthodologie utilisée recourt à une analyse de contenus grâce à une méthode inter-juges. Les résultats de cette recherche permettent de dégager des pistes de réflexion, sur le plan théorique et sur le plan pratique. 


\title{
Dintonestse
}

Volugle 3, numéro 1-2 | janvier-avril 2014

\section{Le stage en formation alternée dans l'enseignement supérieur : pour quel développement professionnel ? Le cas d'une formation universitaire aux métiers de la formation}

\author{
Jérôme ENEAU*Geneviève LAMEUL* Éric BERTRAND**
}

\section{${ }^{*}$ CREAD}

Université Rennes 2

Rue du Recteur Paul Henry

35000 Rennes, France

jerome.eneau@univ-rennes2.fr

genevieve.lameul@univ-rennes2.fr

\author{
${ }^{*}$ REV/CIRCEFT \\ Université Paris Est Créteil \\ 61 avenue Général de Gaulle \\ 94010 Créteil, France
}

Mots-clés : analyse réflexive, portfolio, professionnalisation, développement professionnel, pédagogique universitaire

Résumé: Cette recherche part de l'analyse de dix documents réflexifs, produits en fin d'année dans le cadre de l'accompagnement à la professionnalisation par des étudiants de master 1 et master 2 SIFA (Stratégie et ingénierie en formation d'adultes) de l'Université Rennes 2. Cette production réflexive, qui vise à formaliser et expliciter l'articulation des approches théoriques et praxéologiques caractéristique de ce Master, rend compte du vécu de stage en alternance à visée intégrative, de la part des étudiants. L’analyse de ces écrits permet de caractériser le processus de leur développement professionnel à partir d’un modèle visant à articuler trois dimensions : 1) les dynamiques identitaires en jeu dans ce processus de professionnalisation ; 2) la construction d'une socialisation professionnelle progressive ; 3) lélaboration d'une posture spécifique de praticienréflexif. La méthodologie utilisée recourt à une analyse de contenus grâce à une méthode inter-juges. Les résultats de cette recherche permettent de dégager des pistes de réflexion, sur le plan théorique et sur le plan pratique.

Title : The internship training alternating in higher education: what kind of professional development? The case of a university education for careers in training

Key-words : reflexive analysis, portfolio, professionalization, professional development, higher education

Abstract: This research aims to analyze the professionalization process of Master students in an adult education program, based in the University of Rennes (Brittany). The material uses ten reflexive analysis papers, produced by the students for each end of their year session (Master 1 and Master 2). This reflexive production, which aims to formalize and clarify the articulation of theoretical and praxeological aspects of the Master program, shows the integrative experience acquired during the year. The analysis of these papers allows us to characterize the process of professional development from a model articulating three dimensions : 1) the identity dynamics linked with the process of professionalization, 2) the progressive professional socialization required by this professionalization, 3) the development of a specific posture, as a reflexive practitioner. The methodology employs a content analysis through inter-rater method. The results of this research offer a discussion on theoretical and practical levels, both for a better comprehension of professional development and for the trainers or program managers, to support this process of professionalization. 


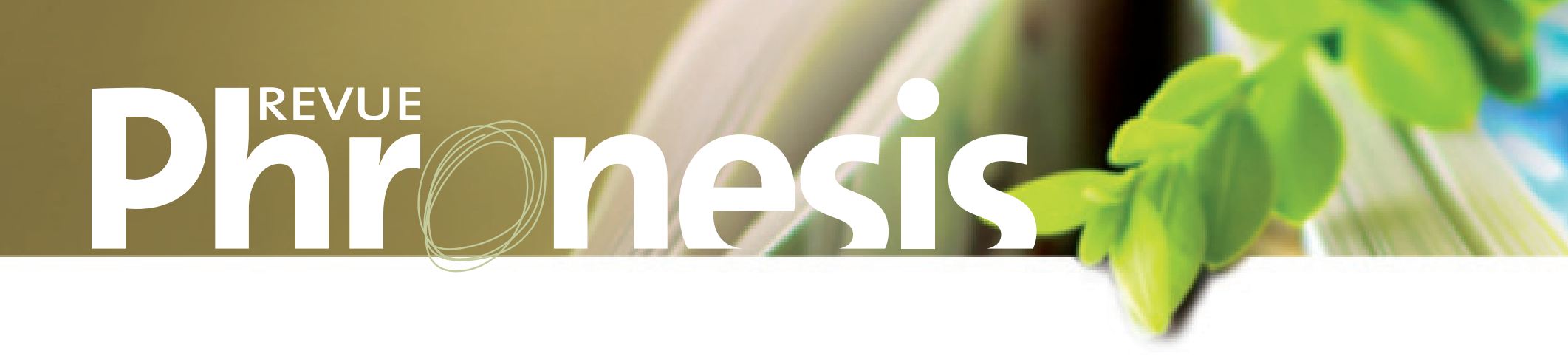

\section{Le dispositif de formation du Master SIFA}

Le dispositif universitaire du master SIFA (Stratégie et ingénierie en formation d'adultes), de l'Université Rennes 2, a pour objectif la professionnalisation aux métiers de la formation. Il accueille vingt à vignt-cinq apprenants par année, soit une cinquantaine de personnes réparties entre master 1 et master 2, dont une majorité de professionnels en reprises détudes. Notre programme poursuit donc des visées d'accès et de préservation de l'emploi, via l'acquisition de compétences sanctionnées par un diplôme, mais il vise aussi laccompagnement des sujets dans leur réalisation personnelle et leur développement, en tant que sujets sociaux (Eneau, Bertrand \& Lameul, 2012).

La formation se déroule en alternance : les apprenants viennent une semaine par mois à l'université, de septembre à juin, et sont « sur le terrain » le reste du temps, sous différents statuts selon les apprenants (stage, contrat de professionnalisation, contrat d’apprentissage, etc.) ; différentes unités de formation sont consacrées, lors des séances de regroupement, à l’analyse de pratiques et à la construction du projet professionnel. Ces unités de formation sont les lieux d'exploration et d'explicitation de l'expérience vécue, utilisant la discussion comme espace de «formation dialogique " (Labelle, 1996; Jacques, 2004). Elles permettent d’aborder de manière réflexive non seulement l'expérience vécue sur les lieux de stages ou d'exercice professionnel mais aussi l'expérience antérieure et les "cadres de références " des étudiants (Mezirow, 2001 ; Brookfield, 2005), dans toute leur singularité et leur diversité.

Parce qu'il permet un certain équilibre entre l'appris, l’expérimenté et le professionnel, le stage est conçu, depuis la création du master', comme un mode pédagogique porteur d'une dimension intrinsèquement formative. Le dispositif d'alternance à visée intégrative s'inscrit dans une logique de formation "par » et « pour » la recherche, favorisant une réflexion « dans » et «sur » l'action. Au delà de l'aspect conventionnel, et même s'il ne donne droit quà gratification et non à rémunération, le stage est considéré comme une période de professionnalisation au même titre que les autres types de formation sur le terrain. Concernant majoritairement des étudiants issus de la formation initiale, il s’adresse aussi, cependant, à des adultes en reprise détude. Comme pour les autres types de contrat, les temps de stage sont imbriqués dans lélaboration d’une problématique de recherche, menée par les étudiants mais accompagnée par un enseignant et un tuteur opérationnel.

À l'université, l'analyse des pratiques représente un lieu de confrontation qui questionne les logiques de la formation, de l'action située et de la recherche à la fois, avec des visées heuristiques de production de savoirs généralisables mais aussi de savoirs plus personnels et plus " expérientiels ". Les conduites des étudiants pendant les travaux individuels et collectifs à l'université au sein des unités de formation dénommées " praxéologie » en première année et « analyse des pratiques » en seconde année, la production des travaux réflexifs demandés dans le cadre de la formation, mais aussi la production individuelle des écrits de fin d’année (dont le mémoire de fin de master 2) sont autant de lieux et de moments où est étudié le « dialogisme expérientiel » (Bertrand, 2007), c'est-à-dire la combinaison de logiques vécues, construites dans les périodes à l'université et sur le terrain, de maîtrise technique, de développement intellectuel, de réflexivité et d'intercompréhension.

Ce master est conçu selon un modèle pédagogique fortement ancré sur la réflexivité critique, mobilisant les perspectives de l’apprentissage transformateur et celles d'auto- et de co-formation (Eneau, Bertrand \& Lameul, 2011). Il vise à former des professionnels capables d'agir avec distance réflexive et jugement critique autant sur les situations de formation qu'ils doivent concevoir qu'à agir sur le monde professionnel auquel ils sont préparés, pour « agir par eux-mêmes », en pleine responsabilité. De ce point de vue, l'utilisation des expériences vécues, au regard de l'alternance sur laquelle est basée la formation, comporte plusieurs enjeux : construire à partir de laction une réflexion sur sa posture professionnelle, se construire une identité ancrée dans l'expérience à la fois personnelle et collective à travers un processus de socialisation professionnelle progressive, construire enfin une réflexion éthique basée sur la distanciation et la mobilisation de savoirs critiques. Le développement professionnel se construit ainsi au sein de l'articulation des différentes modalités de formation et des différents espaces de vie de létudiant. Les dossiers d’analyse réflexive élaborés en fin d’année, qui sont présentés dans cette étude, sont particulièrement révélateurs de la manière dont le développement professionnel sélabore entre terrain de stage et apprentissage universitaire. En effet, alors que nous explorons en parallèle la possibilité de faire évoluer ces dossiers d’analyse réflexive vers une format de " e-portfolio », il apparait d’autant plus important, au plan théorique comme au plan pratique, de questionner la dimension formative de ce travail réflexif dans le développement professionnel des étudiants, au regard de différents questionnements : Existe-t-il une "progressivité » ou non des modes de professionnali-

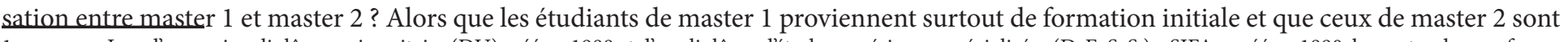

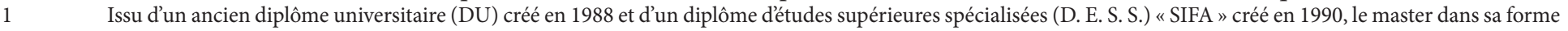

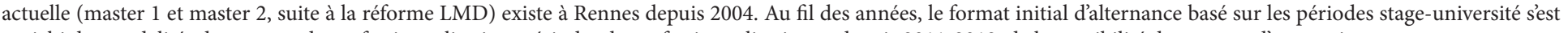
enrichi des modalités de contrats de professionnalisation, périodes de professionnalisation et depuis 2011-2012, de la possibilité de contrats d'apprentissage. 
plutôt issus de formation continue, peut-on identifier, au regard de ces deux populations, des déterminants de leurs « voies de professionnalisation " (Wittorski, 2011) ? Plus largement, alors que certains auteurs avaient annoncé une " déstagification » progressive de la formation (Carré, 1992, p. 31) au profit de modalités "d’autoformation », de formation plus " ouverte » ou " hybride », qu'en est-il de la pertinence de cette " petite forme » pour la professionnalisation actuelle des étudiants? Enfin, et même s'il n’est pas question de procéder ici à une analyse comparative entre leurs différents statuts (stage, contrat d'apprentissage, contrat de professionnalisation, etc.), quel rôle joue le stage en particulier dans le développement professionnel des étudiants de ce Master?

Après avoir procédé à une analyse théorique des notions mobilisées, ce sont ces questions qui vont guider la présente recherche à partir de l'analyse, sur le plan empirique, de dossiers d'analyse réflexive de 10 étudiants-stagiaires de Master 1 et de Master 2. En conclusion, nous présenterons les implications pratiques pour la pédagogie et les enjeux théoriques pour la recherche auxquels aboutit cette analyse.

\section{Stage et formation alternée : pour quel développement professionnel ?}

Partant des questions relatives au stage comme vecteur du développement professionnel des étudiants, nous avons posé l'hypothèse que l'analyse réflexive des pratiques des stagiaires, lors des semaines de regroupement à l'université et en particulier du travail mené en praxéologie, pouvait les amener à construire cette professionnalisation graduellement, tout au long de l'année. Cette hypothèse de travail conduit à préciser au préalable ce qui est entendu ici par "professionnalisation » et « développement professionnel », ainsi que la manière dont le stage et l'analyse des pratiques engagées lors du stage, peuvent participer à ce processus.

\subsection{Professionnalisation et développement professionnel}

À l'heure d'injonctions toujours plus pressantes, pour l'université, à la professionnalisation de ses formations, les ambigüités restent nombreuses et entretiennent depuis de nombreuses années pour les étudiants, pour les enseignants comme pour les milieux professionnels, des attentes paradoxales et des perceptions parfois divergentes sur les missions et les finalités de ces formations de l'enseignement supérieur (Altet \& Bourdoncle, 2000 ; Agulhon, 2007 ; Épiphane et Giret, 2012 ; Maillard, 2012).

Ainsi que le souligne Wittorski $(2007,2008)$, le terme de professionnalisation conserve, dans ses usages comme dans son absence de définition univoque, une variété d’acceptions qui ne favorise pas la levée de ces ambigüités; entre « fabrication de professionnels » et « recherche d’efficacité et de légitimité plus grandes », l’université oscille ainsi entre différentes « voies de professionnalisation ». De même, entre les deux acceptions de la professionnalisation, comme constitution de groupes professionnels d'une part et comme développement professionnel des individus d'autre part, l'université et les formations aux métiers de la formation auxquelles elle prépare participent par ailleurs à entretenir la confusion, tant la définition des métiers, des fonctions et des activités échappe aux différents acteurs (universités elles-mêmes mais aussi organismes de formation et entreprises, individus et groupes professionnels exerçant dans le milieu de la formation, etc.) (Brémaud \& Guillaumin, 2010 ; Eneau \& Rey, 2011). Enfin, entre les « enjeux sociaux » et la " charge idéologique » que le terme porte intrinsèquement (Wittorski, 2008 ; Eneau, 2011), la professionnalisation reste au cœur de tensions axiologiques qui nous font préférer aujourd'hui, en tant que responsables de formation aux métiers de la formation, le choix du terme de " développement professionnel » des étudiants dont nous avons la charge dans le master SIFA, puisqu'ils sont pour la plupart des adultes possédant déjà une expérience professionnelle et ne sont donc pas stricto sensu «à professionnaliser ».

La question du développement professionnel de ces apprenants adultes n’est toutefois guère plus aisée à définir. Certaines études anglophones sur le développement professionnel dans le monde de l'éducation (Day, 1999 ; Fassler et Christensen, 1992 ; Garet et al., 2001 ; Pratt, 1998) soulignent que ce développement est lié à des valeurs et un engagement personnel, qu'il se produit dans et par l'action, avec et par les pairs, mais qu'il est lié aussi à la construction d'une « pensée critique » et une certaine forme "d'intelligence émotionnelle »; il permet d'articuler des connaissances théoriques et des savoirs procéduraux, de prendre conscience de ses pratiques et des cadres de références qui influencent la prise de décision, en fonction des problèmes rencontrés. Selon ces auteurs, ce développement se construirait par phases successives, à la fois processus et produit de la formation initiale et continue, mais garantirait in fine une "pratique de qualité », reconnue sur le plan professionnel.

Plus récemment, certains travaux francophones sur le développement professionnel ont permis par ailleurs de souligner l'influence du dispositif de formation sur le développement identitaire des apprenants ainsi que le lien entre les caractéristiques du dispositif et les "pratiques professionnelles de référence », mentionnant notamment les liens existants entre développement professionnel, socialisation professionnelle et « effet-enseignant» (Charlier, Nizet \& Van Dam, 2006 ; Martineau, Presseau \& Portelance, 2009 ; Lameul, Eneau, Charlier et al., 2011). Deux séries d'études conduites dans l'enseignement supérieur nous ont ainsi amenés, dans des contextes différents, à relever les incidences de l'apprentissage non formel et informel sur le développement professionnel (Lameul, Eneau, Charlier et al., 2011) et la place d'un apprentissage " transformateur ", dans la prise de conscience de ces pratiques professionnelles (Eneau, Bertrand \& Lameul, 2011 ; 2012). Comme ces études ont pu le montrer, le développement résulte alors d’un double processus d’autoformation, dans une perspective «identitaire » de développement de soi, et de co-formation, dans une perspective de «socialisation » par les pairs et par l'environnement professionnel. 


\subsection{Développement professionnel ou construction de soi?}

Lensemble de ces travaux, au delà des ambigüités conceptuelles déjà soulignées pour le terme de professionnalisation, permet d'identifier certaines dimensions constitutives du développement professionnel qui peuvent être rapprochés des « six voies de la professionnalisation ${ }^{2} »$ identifiées par Wittorski (2012) dans les formations universitaires professionnalisantes : (1) le développement de capacités ou d'habiletés, mises à lépreuve des pratiques d'un milieu, confrontées aux compétences attendues par l’environnement professionnel et qui peuvent être rattachées à la notion de "genre ", proposée par Clot (2000); (2) le développement identitaire, dans une double dimension à la fois personnelle et professionnelle, qui rappelle à quel point ce développement est construit par soi mais aussi « par » et " grâce à » autrui (Kaddouri et al., 2008) ; (3) un développement intrinsèquement " processuel », enfin, soulignant l'articulation permanente entre réflexion et action, mais aussi une évolution continue et une remise en cause toujours possible.

Plus que dans les travaux portant spécifiquement sur la professionnalisation, ces dimensions du développement professionnel se retrouvent aujourd'hui dans les travaux s'intéressant à la construction identitaire (personnelle et professionnelle, individuelle et collective) et à la construction de la professionnalité (comportant elle aussi une double dimension identitaire et collective), certes dans une logique de construction de compétences pour l'apprenant, mais aussi de reconnaissance et de légitimation, tant sociales que professionnelles (Beckers, 2007 ; Jorro, 2009 ; Jorro \& De Ketele, 2011 ; Kaddouri, 2008, 2010). Ce que permettent de relever ces travaux, ce sont non seulement les différentes facettes de la construction identitaire, pour l'individu, dans des composantes à la fois cognitives et opératoires, représentationnelles, socialement construites et donc évolutives, mais aussi émotionnelles, socioaffectives et donc non totalement prédictibles. De même, la professionnalité se construit dans une temporalité qui incite à appréhender lévolution du « soi professionnel » comme processus dynamique, puisque la construction intermédiaire vécue par le sujet se formant s’appuie sur des savoirs génériques propres au métier visé et sur des savoirs singuliers, sans lesquels le stagiaire ne pourrait pas se reconnaitre dans son action ou ses gestes professionnels (Jorro \& De Ketele, 2011). La question de " léthos professionnel " (Jorro, 2009) prend ici toute son importance, dès lors que le sujet déclare travailler sur sa posture qui reste, lors de la formation, encore en construction3 ; le professionnel « se formant » vit en effet des processus de transformation prenant appui sur des savoirs théoriques, des savoirs d’action, mais aussi sur une réflexion éthique.

La question du repérage des éléments participant à construire cette professionnalisation en cours de construction, ce développement professionnel ou cette professionnalité émergente devient alors un pari risqué, puisqu’lle nécessite de lire des "processus intermédiaires », des embryons d'action, l'esquisse de savoirs professionnels (Jorro \& De Ketele, 2011) : comment aborder l'analyse de gestes encore peu assurés, de compétences en cours de construction, d'identités en transition, d'intentions encore peu explicites ? Une approche de lentre-deux, du cheminement et du transitoire est en jeu, conduisant à privilégier, sur le plan empirique, le repérage de traces indiciaires de ce développement professionnel en cours, à la manière dont Jorro et De Ketele (ibid.), par exemple, proposent de relever des traces de professionnalité, par une " compréhension indicielle de l'agir ", en croisant des indices stratégiques, tactiques et éthiques, relevant tant du geste éphémère, de l’action passagère, de marques d'expertise, que de la dynamique identitaire qui s'affirme autour des valeurs et des normes du métier. De ce point de vue, l'analyse des portfolios proposée par Jorro (ibid.) offre une voie intéressante pour le repérage de ces « traces » de la professionnalité émergente.

\subsection{Rôle du stage, alternance et accompagnement des stagiaires}

Les enjeux d'identification et de repérage des traces de ce développement professionnel ouvrent ainsi un double défi. Au plan théorique, il s'agit tout d'abord d'offrir un construit qui permette d'en appréhender les différentes dimensions ou composantes (identitaires et donc individuelles), concernant le développement des apprenants. Au plan pratique, ce cadre doit aussi correspondre à la fois au dispositif de formation existant (formation en alternance, se déroulant sur deux ans, avec des publics différents, etc.) et aux enjeux pédagogiques liés à l’accompagnement des stagiaires pour en améliorer les pratiques.

Dans la lignée de travaux antérieurs, portant sur les apports d'une démarche de "formation-action-recherche " pour la pédagogie universitaire (Eneau, Bertrand, Lameul, 2011 ; 2012), nous avons fait le choix de partir ici d'une démarche inductive, articulant deux sources d'inspiration : ont été pris en compte, d'une part, les acquis de l'expérience des auteurs de cette recherche, en tant que responsables pédagogiques d'unités de formation d’analyse de pratiques et accompagnant les stagiaires dans leurs différentes « voies de professionnalisation » (Wittorski, 2012) et d'autre part les résultats des recherches anglophones et francophones présentées ci-dessus. Cette articulation nous permet de retenir plus particulièrement trois des différentes dimensions « processuelles » du développement professionnel :

1. la construction ou la transformation identitaire, par soi-même et par les groupes sociaux d'appartenance, repérables à travers les « dynamiques » et les « tensions » identitaires, telles quétudiées par Kaddouri $(2002 ; 2006 ; 2008)$ notamment, et déclarées par les stagiaires à travers des situations ou des évènements significatifs pour eux ;

2. les différentes formes de socialisation professionnelle, issues à la fois des acquis du stage sur le terrain et de l'analyse réflexive effectuée à l'université, dont les effets peuvent être repérés dans les formes de socialisation mises en exergue dans les travaux de Dubar (2010) et opérationnalisées de manière particulièrement significative par Beckers (2007) ou encore Martineau, Portelance et Presseau (2009);

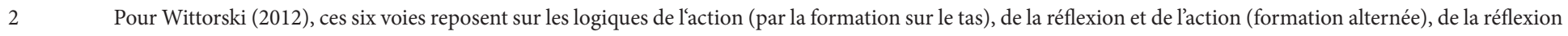

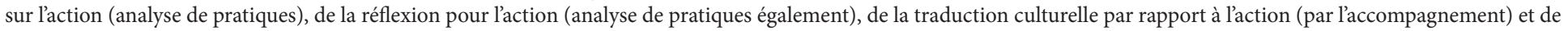
l'intégration-assimilation (à travers la formation magistrale).

3 Ce qui conduit Barbier, par exemple, à parler de « pré-professionnalisation " pour les formations dites « professionnalisantes », à l'université. 
3. la construction d'une posture et de compétences spécifiques, identifiées par le stagiaire et donc toujours éminemment personnelles, mais reconnues aussi comme légitimes par le milieu professionnel, l'ensemble de ces compétences et des traces indiciaires de cette posture et de cet éthos étant repérables à travers les caractéristiques identifiées dans les travaux de Lameul $(2006,2011)$ et Jorro $(2009$; 2011) notamment.

Ce cadre offre ainsi un point de départ pour l'analyse des discours produits pas les étudiants au sujet de leur propre développement professionnel. Comme toute catégorisation, il revêt une certaine artificialité dans la mesure où les dimensions de construction identitaire et de socialisation sont des processus étroitement imbriqués, comme l'ont relevé les auteurs mentionnés supra à la suite de Dubar, dont ce cadre est inspiré. Il offre cependant l'avantage, au plan théorique, de pouvoir procéder à une analyse catégorielle à partir du verbatim produit par les étudiants à propos de leur professionnalisation. Il offre de plus une grille de lecture des processus participant à la professionnalisation et au développement professionnel, d’un point de vue pratique, plus fine que celle offerte par les définitions ambigües relevées dans la littérature. En reprenant les dimensions de la construction identitaire, de la posture professionnelle et de la socialisation et l'acculturation professionnelles graduelles, à travers le stage et l'analyse réflexive produite lors des séances à l'université, cette catégorisation vise aussi à fournir des indicateurs précieux sur le plan pédagogique. En effet, une telle méthode de repérage des processus à l'œuvre peut savérer féconde sur le plan de l'accompagnement des stagiaires, dans la mesure où la modalité d’alternance sur deux années, dans le Master SIFA en particulier, est une voie de professionnalisation articulant les savoirs issus de l'expérience acquise à la fois par le travail, sur le lieu de stage (Liaroutzos, 2012; Mayen, 2012 ) et à la fois à l'université, par soi mais aussi par les autres (Crochard, 2007 ; Fernagu-Oudet, 2007 ; Zaouani-Denoux, 2007). Ainsi, les catégories de la construction identitaire, de la socialisation professionnelle et de la posture professionnelle offrent-elles un cadre d’analyse opérationnel pour appréhender le développement professionnel détudiants-stagiaires, dans une formation professionnalisante aux métiers de la formation, à l'université.

\section{Méthodologie de recueil et d'analyse des données}

Afin d'examiner le rôle et la place du stage dans le développement professionnel des étudiants du master SIFA, nous avons choisi d'examiner plus particulièrement les documents réflexifs produits par ces étudiants en fin de première et de seconde année. Ces documents sont demandés par les deux enseignants accompagnant l'unité de formation " praxéologie » en master 1 et « analyse des pratiques » en master 2, sous une forme similaire pour les deux années (dossier d'une quinzaine de pages, traitant d'une thématique particulière choisie par létudiant, abordée par exemple lors d'une des séances mensuelles d’analyse des pratiques mais pouvant résulter d’un choix plus personnel : situation vécue en stage, analyse réflexive du parcours de l’année, analyse distanciée d’une situation critique, etc.).

Dans le cadre de cette recherche, nous avons procédé à l'analyse qualitative de dix dossiers détudiants ayant un statut de "stagiaires » (cinq étudiants de master 1 ; cinq étudiants de master 2), sachant que parmi l'ensemble des étudiants (plus d'une vingtaine pour chaque année de master), certains effectuent leur formation sous d'autres statuts (contrat d’apprentissage, contrat de professionnalisation, congé individuel de formation, emploi fixe).

Construit à partir du travail exploratoire qui vient dêtre présenté, notre cadre d’analyse mobilise donc plus particulièrement trois des dimensions processuelles du développement professionnel : construction identitaire, socialisation professionnelle, développement d'une posture professionnel, etc. Cependant, la démarche itérative qui a conduit à construire ce cadre a été progressivement enrichie et amendée, au fur et à mesure de l'analyse des premiers « dossiers réflexifs », amenant ainsi les auteurs, dans une démarche intersubjective de confrontation de leurs propres références, à affiner les catégories initialement tirées des écrits scientifiques. ${ }^{4}$ Ces catégories principales, repérées dans la littérature ont alors été modifiées ou complétées, le cas échéant, par des critères émergeant lors de l’analyse elle-même5.

La catégorisation finale a permis de regrouper les dimensions et critères suivants :

\section{Développement d'une posture professionnelle}

1.1. Croyances et valeurs

1.2. Intentions

1.3. Actions

1.4. Éthique professionnelle

2. Construction identitaire

2.1. Tensions/dynamiques identitaires

4 Par exemple, Kaddouri (2010) propose une typologie des dynamiques identitaires : de transformation (changer d'identité) ; d'entretien (entretenir en la prolongeant une identité existante) ; de préservation (défendre une identité menacée); de confirmation (faire reconnaitre une identité nouvellement acquise et insuffisamment reconnue par un " autrui significatif»); de redéfinition (redéfinir une identité floue) ; Martineau, Portelance et Presseau (2009) proposent quant à eux une catégorisation de la socialisation professionnelle en termes de : maitrise de compétences ; développement de relations sociales ; acculturation ; maitrise du " jargon " professionnel ; capacité à utiliser les structures de pouvoir formelles et informelles; connaissance historique de lorganisation.

5 Par exemple, pour la dimension «identité professionnelle », nous avons été amenés à produire une catégorie « s’affirmer/prendre position », qui a émergé de l’analyse de contenu comme catégorie distincte de celles initialement prévues; de même, la catégorie "éthique professionnelle " a émergée comme catégorie liée à la dimension " posture professionnelle ", alors qu’elle nétait pas envisagée comme telle au départ ; à l'inverse, la catégorie "appartenance ", dans la dimension "socialisation ", était prévue au départ pour le codage mais s’est révélée très peu représentative ( 1 codage sur les 5 premiers dossiers); elle a été regroupée avec la catégorie « culture professionnelle/acculturation ». 
2.2. Projet (de soi pour soi/d'autrui pour soi/de soi pour autrui)

2.3. Transformation identitaire (de soi/par rapport à autrui)

2.4. Reconnaissance (de soi/par autrui)

2.5. S’affirmer / prendre position (par soi-même/par rapport à autrui)

2.6. Destructivité / négativité (doutes et dilemmes/remise en cause de soi)

3. Socialisation professionnelle

3.1. Culture professionnelle et acculturation

3.2. Rencontres et échanges

3.3. Appartenance

3.4. Développement de compétences / maitrise de gestes professionnels / genre

Après différentes phases de concertations successives, aboutissant à cette catégorisation commune et progressivement stabilisée, les trois auteurs ont ensuite effectué chacun séparément l’analyse des dix dossiers d’étudiants-stagiaires, étant entendu que la consigne de « l’analyse de contenu » devait respecter les critères d'exclusivité et d'exhaustivité de toute catégorisation (Bardin, 2007). Lanalyse a donc été effectuée grâce à une méthode "inter-juges » (Chi, 1997 ; Kerlinger et Lee, 2000), déjà employée par ailleurs pour l’analyse de contenu de documents réflexifs (Eneau et Develotte, 2012 ; Kuster et Lameul, 2011). Concrètement, cette méthode consiste pour chacun des « juges » (ici les auteurs de l'article, en " triple aveugle ») à catégoriser chaque dossier à partir d'une grille commune, puis de demander la validation ou l'invalidation par les deux autres juges, laccord final d’au moins deux juges sur trois étant nécessaire pour retenir la catégorisation comme « valable » (voir exemple Tableau 1).

\section{Tableau 1}

Extrait de la catégorisation « Construction Identitaire - Étudiants de M1 »

\begin{tabular}{|c|c|c|c|c|}
\hline Étudiant & Juge 1 & Juge 2 & Juge 3 & Extrait codé \\
\hline Mme C. (M1) & $\begin{array}{c}2 \cdot 4 \\
\text { Reconnaissance }\end{array}$ & (non codé) & $\begin{array}{c}2 \\
\text { Reconnaissance }\end{array}$ & $\begin{array}{l}\text { "Mon projet a en outre été validé } \\
\text { par d'autres supérieurs }(\ldots) \text { et permet } \\
\text { d’améliorer ma vision concernant ma } \\
\text { pratique professionnelle et de gagner en } \\
\text { légitimité au sein de la structure. » }\end{array}$ \\
\hline
\end{tabular}

Cette méthode qualitative d'analyse de contenu vise plus, dans ce cas, à obtenir un consensus sur la catégorisation à partir d'un processus d'intersubjectivité (entre juges) quà fournir des données prétendant à lobjectivité (Chi, 1997). Les résultats de cette catégorisation peuvent toutefois être exploités tant dans leurs dimensions quantitatives que qualitatives, le biais de la production des discours à partir d'injonctions des juges eux-mêmes (ici les enseignants référents des unités d’enseignement ayant demandé la production des documents réflexifs analysés), étant alors partiellement neutralisé (Eneau et Develotte 2012).

4. Résultats de l’analyse des dossiers réflexifs et interprétation

4.1 Sur le plan quantitatif

Dix dossiers d'étudiants-stagiaires ont été analysés ( 5 de M1, 5 de M2), représentant un total de près de 37500 mots (environ 20000 mots pour les dossiers de M2, 15000 mots pour les dossiers de M16). 199 extraits ont été codés pour les dossiers de M1 et 228 pour les dossiers de M2 (soit en tout 427 extraits, sur le modèle du tableau 1), représentant respectivement $54 \%$, pour les M1, et $62 \%$, pour les M2, du verbatim total produit par les étudiants dans les dossiers analysés. Le détail du codage effectué est présenté dans le tableau 2, ci-dessous : $6 \quad$ Malgré une consigne identique, les dossiers des étudiants de M2 sont en effet légèrement plus longs que ceux de M1 (environ 4000 mots en moyenne pour les M2,
3500 pour les M1). 
Tableau 2

Codage des 10 dossiers après accord « inter-juges »

\begin{tabular}{|c|c|c|}
\hline & Dossiers de M1 & Dossiers de M2 \\
\hline Posture professionnelle & $\begin{array}{c}56 \text { extraits codés } \\
40 \text { font accord (soit } 71 \% \text { d'ac- } \\
\text { cord entre juges) } \\
14 \text { sur croyances } \\
11 \text { sur action } \\
9 \text { sur intentions } \\
6 \text { sur éthique prof. }\end{array}$ & $\begin{array}{c}52 \text { extraits codés } \\
39 \text { font accord (soit } 75 \% \text { d'ac- } \\
\text { cord entre juges) } \\
17 \text { sur croyances } \\
7 \text { sur action } \\
5 \text { sur intentions } \\
5 \text { sur éthique prof. }\end{array}$ \\
\hline Construction identitaire & $\begin{array}{c}78 \text { extraits codés } \\
55 \text { font accord (soit 70,5\% } \\
\text { d'accord entre juges) } \\
12 \text { sur tensions/dynamiques } \\
2 \text { projet } \\
8 \text { transformation } \\
13 \text { reconnaissance } \\
15 \text { s'affirmer, prendre position } \\
5 \text { destructivité/négativité }\end{array}$ & $\begin{array}{c}93 \text { extraits codés } \\
81 \text { font accord (soit } 87 \% \text { d'ac- } \\
\text { cord entre juges) } \\
13 \text { sur tensions/dynamiques } \\
11 \text { projet } \\
34 \text { transformation } \\
8 \text { reconnaissance } \\
9 \text { s'affirmer, prendre position } \\
10 \text { destructivité/négativité }\end{array}$ \\
\hline Socialisation professionnelle & $\begin{array}{c}\text { 65 extraits codés } \\
45 \text { font accord (soit } 69 \% \text { d'ac- } \\
\text { cord entre juges) } \\
5 \text { sur culture prof/accultur } \\
25 \text { sur rencontres/échanges } \\
15 \text { sur développement comp. }\end{array}$ & $\begin{array}{c}83 \text { extraits codés } \\
70 \text { font accord (soit } 84 \% \text { d’ac- } \\
\text { cord entre juges) } \\
14 \text { sur culture prof/accultur } \\
41 \text { sur rencontres/échanges } \\
14 \text { sur développement comp. }\end{array}$ \\
\hline
\end{tabular}

Dans ce tableau, la totalité des extraits ayant fait accord entre deux juges au moins n’est pas toujours égale à la somme des critères caractérisant la dimension examinée ; en effet, des codages initiaux d'un des juges ont pu être requalifiés par les deux autres dans des catégories différentes ${ }^{7}$ Par ailleurs, les pourcentages d’accords sont globalement plus élevés pour les dossiers de M2, les confrontations successives pour stabiliser la catégorisation ayant bénéficié aux dossiers de M2, analysés à la suite des dossiers de M1.

Malgré ces biais méthodologiques, les grandes tendances résultant de ce travail de catégorisation restent significatives. Une première remarque peut être établie au sujet de questions posées initialement, concernant les éventuelles différences entre M1 et M2 et résultant d'un accompagnement différent, par deux enseignants privilégiant potentiellement des approches elles aussi différentes. Or, il ressort de ces premiers résultats que dans les deux cas, c'est d’abord la dimension « construction identitaire » qui est majoritairement citée ( $39 \%$ pour les M1, $41 \%$ pour les M2), suivie dans les deux cas par la dimension «socialisation» (33\% et $36 \%)$ et enfin par celle de " posture» $(28 \%$ et $23 \%)$. L’effet-enseignant anticipé (l’enseignant « orientant » les réponses) n’est donc pas confirmé.

\subsection{Auplan qualitatif}

C'est à l'intérieur de chacune des dimensions constituées en catégories que les items marqueurs du développement professionnel, mis en avant par les étudiants, peuvent ensuite être différenciés.

Si le travail réflexif demandé en fin de M1 comme de M2 reflète d’abord une préoccupation identitaire, de la part des étudiants, ce sont les items «s'affirmer / prendre position » et « reconnaissance » qui ressortent des dossiers de M1, alors que c'est celui de « transformation » qui ressort pour les M2. Pour les étudiants de M1, le stage est plus souvent une opportunité de construction identitaire dans un sens positif, 
permettant de poser graduellement des actes reconnus sur le plan professionnel, de gagner en légitimité aux yeux des collègues de travail. Ainsi, une stagiaire de M1 affirme : «Construire son identité professionnelle, c'est s'investir dans des relations durables afin d’obtenir une reconnaissance mutuelle des co-acteurs... le fait que (...) mes collègues ont validé ce projet m’assure que je suis en mesure d’avoir une représentativité au sein de la structure (...), que j’ai acquis une certaine légitimité. » Cette construction identitaire passe aussi par des " tensions », des « dynamiques » qui ne sont pas toutes positives, ramenant létudiant à son statut d’apprenant, pas tout à fait encore un «professionnel » comme les autres : «Bien qu’elle soit très satisfaite du travail que j’ai pu accomplir, (ma tutrice) m’a conseillé de me repositionner en tant que stagiaire».

À l'inverse, pour les étudiants de M2, cette construction passe avant tout par un processus de «transformation » qui reflète des «tensions » et des « dynamiques » plus fréquemment négatives. Ayant acquis plus souvent que les étudiants de M1 une expérience préalable (et donc probablement une identité professionnelle déjà plus affirmée), les étudiants de M2 se trouvant en position de stagiaires soulignent aussi plus fréquemment des ressentis négatifs, sur le plan identitaire, comme cette étudiante de $\mathrm{M} 2$ : « Ma position de stagiaire (...) ne me fait rentrer dans aucune des cases et génère de l'incompréhension. Je ne peux pas avoir d'identifiant, pas faire de déplacements, pas me connecter de chez moi à mon adresse professionnelle...». Une autre stagiaire de M2 raconte : «la mise en situation professionnelle m’a amené non sans souffrance à la négociation de mes intérêts premiers (...) la confrontation de deux mondes (...) a bouleversé profondément mes idéaux et mes aspirations. Ma perception de la déshumanisation du travail a mis à mal mes convictions premières ». "Je suis très vite entrée, raconte encore cette autre étudiante, dans ce que j’appellerai le «complexe du stagiaire». (...) Quelle est ma place ? Comment accepter que l'entreprise soit un lieu où je ne «sais pas» ? " ... Les deux cas, M1 comme M2, illustrent donc bien comment les processus « tensionnels » de la dynamique identitaire (Kaddouri, 2002 ; 2008) sont à l’œuvre dans la construction professionnelle de l'ensemble des stagiaires.

Concernant la seconde dimension du cadre d’analyse qui apparaît dans les dossiers des étudiants-stagiaires, la « socialisation professionnelle » se produit le plus souvent à travers des « rencontres » et des « échanges » qui participent au développement professionnel, en particulier pour les étudiants de M2. Cette socialisation passe tant par l'immersion dans un terrain de stage (et donc par les collègues de travail), que par le groupe détudiants à l'université (notamment lors de l’analyse de pratiques). Une étudiante de M2 relève ainsi : « Le co-développement qui sest avéré le plus pertinent et efficient pour moi est celui que j’ai pu instaurer avec mon collègue et tuteur lors de nos réflexions mutuelles et respectives quant au bon déroulement, à l'avancée et à l'amélioration du dispositif »; une autre que "les séances (à l'université) m’apparaissent alors comme pertinentes pour m’aider à prendre ce recul nécessaire à ma professionnalisation » ; une troisième encore : « Les temps déchange avec la promotion mont permis de dépasser mes a priori, ma conception de la situation et mûrir ma réflexion sur ma pratique. " S'il paraît délicat de différencier quel type déchanges et de rencontres influence le développement professionnel des étudiants, notamment entre M1 et M2, il paraît clair, tout au moins, que le travail d'articulation réflexive entre terrain de stage et analyse de pratique, à l'université, répond aux objectifs d’alternance « intégrative » visés par le dispositif du master.

En second lieu, c'est le développement de compétences et la maitrise d’outils ou de gestes professionnels reconnus par le milieu qui semble refléter, pour les étudiants-stagiaires, des items marqueurs significatifs de leur professionnalité émergente. Par exemple, cette étudiante de M1 raconte : «Les constats faits sur mes pratiques professionnelles (...) mont permis délaborer une grille d'entretien plus approfondie que celle que j’avais construite jusqu'alors (...); j'utilise désormais un outil de diagnostic pour les entretiens. » Pour une autre étudiante de M2, ce sont les deux années de formation qui lui « ont permis d’acquérir, dans un premier temps, une connaissance large des dispositifs actuels (...). J’ai intégré la législation, les nombreux sigles du jargon des professionnels du milieu (...). J'ai donc pu cultiver une certaine expertise du contenu (...). Cela à travers l'immersion quotidienne dans le milieu et notamment par le suivi des actions en cours. » Ceci se traduit ensuite par des réalisations confiées à une stagiaire qui est finalement considérée comme une "professionnelle » par les collègues de son lieu de stage : "J’ai contribué concrètement à la mise en œuvre du Forum pour l'emploi (...). J’ai participé aux différentes étapes de mise en ouvre du projet, des phases d'anticipation, de planification (...), en passant par la mobilisation des acteurs, le lancement, ou encore la gestion des objectifs, des enjeux, des priorités et des risques. » Sans différence significative entre étudiants de M1 ou de M2, ce serait donc d’abord les échanges et les rencontres, à la fois sur le terrain de stage et dans l'analyse conjointe et collective de pratiques, à l'université, qui semblent favoriser une socialisation professionnelle progressive (Martineau, Presseau et Portelance, 2009 ; Dubar, 2010). Les « marqueurs » du professionnalisme, à travers les compétences développées, les tâches confiées ou les gestes professionnels maîtrisés (Clot \& Faïata, 2000 ; Beckers, 2007) n’agissent que secondairement, comme « indices » de la professionnalité émergente (Jorro \& De Ketele, 2011).

Enfin, pour la troisième dimension " construction d'une posture professionnelle », constituée en catégorie de notre cadre d'analyse, elle semble être encore majoritairement soutenue par des représentations, des " croyances » et des " valeurs », pour les étudiants-stagiaires des deux promotions, et de manière surprenante de la part des M2 plus encore que des M1. L'une des étudiantes de M1 se projette ainsi dans l'avenir : « Demain, nous serons des professionnels, dans une position différente de celle d’aujourd'hui. Nos erreurs et nos piétinements ne seront plus couverts par le statut de stagiaire, et le marché du travail ne nous fera pas de cadeau. Nous sommes censés être efficaces et rentables ». À l'inverse, une étudiante de M2 revient sur son parcours antérieur et son statut de stagiaire : « Dans le monde de l'entreprise, ce «statut» m’a très vite déconvenue. Si stagiaire signifie «en situation d’apprentissage», il est très vite associé à la posture du «novice». Novice dans le domaine actuel peut-être, voire sûrement. Mais signifie-t-il pour autant novice dans la vie active ? Dans la vie en général ? " Cette même étudiante affirme : "Ce sont mes croyances en l'humain, en la relation d'aide et mes convictions de pouvoir changer les choses (...) qui mont amené jusqu'ici ; (mais) si cette position de stagiaire me permettait d’observer, elle réduisait tout autant mes possibilités dêttre mobilisée par des acteurs externes à ma structure d'accueil ». À l'instar de la dynamique de transformation identitaire relevée précédemment, ces croyances 
et ces représentations sont ainsi malmenées, chez certains étudiants de M2 : «Pour moi, devenir "quelqu’un" à lépoque passait forcément par l'ascension sociale que je croyais être une véritable reconnaissance. Si je dis "que je croyais", c'est parce que mes représentations ont bien changé depuis. » Ou encore, pour une autre M2 : « C’est davantage ma vision utopique du monde de la formation qui est en balance, dans le sens où l'idée que je m’en étais faite au départ (n’est) pas forcément en adéquation avec ce que j’en vois. »

Nous avions défini ici la "posture professionnelle ", à la suite de Lameul (2006), en tant "quétat mental façonné par les croyances et orienté par les intentions " (notamment en matière d'apprentissage), ce à quoi l'on croit et ce qu’on a l'intention de faire, "donnant sens et justification à l'action ». Les dossiers d'analyse réflexive des étudiants-stagiaires de M1 comme de M2 montrent bien à quel point cette posture se développe au prix d'une remise en cause des représentations antérieures (potentiellement traductrices de croyances et valeurs) et des projets initiaux (traducteurs des intentions). Ainsi, arrivée au terme de sa formation, une étudiante de M2 affirme : « Je souhaite prendre en main mon projet et en faire une opportunité ». Une autre explique la manière dont elle a construit sa posture, pendant l'année de $\mathrm{M} 2$ : « Ma motivation, le sens que je donne à la formation nest pas de seulement appliquer des procédures mais de pouvoir y exercer une certaine ingéniosité. Ancrée dans une éthique et des valeurs humaines fortes (...) je souhaite mettre en adéquation ma posture personnelle avec ma posture professionnelle ». À l'articulation entre croyances et valeurs, intentions et actions, la posture révèle les difficultés de stabiliser un " éthos professionnel» (Jorro, 2009), entre construction et remise en cause identitaire, socialisation et acculturation professionnelle, développement de savoir-faire et de gestes reconnus par le milieu. Comme le résume une étudiante de M2: «Si cette position de stagiaire me permettait d’observer, elle réduisait tout autant mes possibilités dêtre mobilisée par des acteurs externes (...). Je dirais qu'elle était pour moi une entrave à ma socialisation professionnelle, nécessaire à l'acquisition d'une posture et d'une identité professionnelle. De même qu’elle a $\mathrm{pu}$, en certains cas, remettre profondément en cause ma propre identité personnelle ».

\section{Conclusion et ouvertures}

Si les résultats de cette recherche, avant tout qualitative et n'explorant pas la totalité des modes possibles de développement professionnel, ne sauraient être représentatifs de tous les étudiants se formant aux métiers de la formation, à l'université, ils permettent toutefois d’en tirer certaines pistes de réflexion, sur le plan théorique comme sur le plan pratique.

Au niveau théorique

Comparativement à d’autres travaux, ces résultats empiriques montrent que le développement professionnel n’agit pas toujours sur un mode de progressivité mais, quau contraire, le statut de stagiaire, notamment lors d'un retour à l'université après une expérience professionnelle antérieure, peut être fortement déstabilisant sur le plan identitaire, amenant à reconstruire une posture professionnelle elle aussi instable. Cette posture professionnelle peut sembler logiquement plus interrogée en master 1 qu’en master 2 , si l'on considère que ces étudiants plus jeunes, plus fréquemment en formation initiale de surcroit, sont encore en phase de découverte des métiers auxquels ils se préparent. À l'inverse, la place des croyances et des valeurs, mise en exergue par les étudiants de M2, montre la prégnance de cet item dans la construction d'une posture qui interroge toute la fragilité d'un « éthos professionnel » qui serait paradoxalement plus difficile à construire, lors d'un stage, à partir d'une expérience antérieure et d'une reconversion, lors d'une entrée directe en M2. Des recherches complémentaires seraient ici à mener, d'une part pour confirmer ou infirmer ces constats pour un plus grand nombre détudiants, si possible pour différentes formations professionnalisantes, à l'université, et d'autre part pour affiner le cadre d'analyse employé. En effet, en l'absence d'une définition univoque du développement professionnel, nous avons choisi ici de construire un modèle tridimensionnel prenant en compte les processus de construction identitaire, de socialisation et d'élaboration d'une posture professionnelle ; celui-ci reste encore à conforter sur le plan épistémologique et empirique. Au vu des résultats obtenus, sa force heuristique réside dans l'approche réflexive qu'il privilégie et les dynamiques (personnelles et collectives, par soi et par autrui, etc.) qu'il met en exergue, en particulier dans les dimensions temporelles à l’ouvre dans le processus global de développement professionnel, mais que l’analyse de dossiers rédigés en fin d’année ne montre pas dans toute leur totalité et leur complexité. Il pourrait alors être confronté à d’autres approches plus qualitatives, examinant le récit de formation et des dimensions plus biographiques, par exemple, ou plus quantitatives, comparant les dimensions opératoires relevées dans la littérature pour les dynamiques identitaires, par exemple, ou au regard des déterminants de la socialisation professionnelle.

Nous notons un certain nombre de limites de notre travail qu'il importera de prendre en compte dans une prolongation de cette recherche exploratoire : l'usage d'un outil plus spécialisé (par exemple, Alceste) permettrait de traiter plus exhaustivement le contenu de nos données $^{8}$ la prise en compte des profils des étudiants est certainement importante, voire indispensable, pour éclairer la question qui est ici abordée. Dans la perspective d'un approfondissement de cette recherche, notre modèle d'analyse méritera dêtre repris pour questionner tout particulièrement l'articulation et/ou l'emboîtement superposé des trois dimensions processuelles du développement professionnel retenues pour notre étude.

Au niveau pratique

Ces résultats ont aussi plusieurs incidences pratiques, au delà du fait d’amener les auteurs, impliqués par leurs responsabilités pédagogiques dans le développement des étudiants, à confronter leurs propres représentations sur le rôle professionnalisant du stage et de l'analyse des pratiques concomitante. Une telle recherche incite par exemple à revoir la progressivité annoncée entre Master 1 et 2, la constitution des

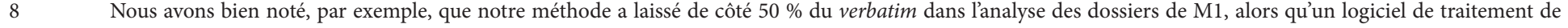
données permettrait un traitement plus systématique, fournissant probablement aussi des résultats complémentaires. 
promotions avec des entrées directes possibles en M2 (traditionnellement importantes car héritières des anciennes « reprises détudes » de D.E.S.S.) ou encore les modalités offertes de stages, pour ces M2 en reprise d'étude, potentiellement plus déstabilisantes que d’autres formes d'alternance (congé de formation, période de professionnalisation, etc.). Pourtant, ce que montrent aussi les résultats de cette étude, c'est bien que le stage, "petite forme » de professionnalisation qui nest pas mineure, reste une modalité intrinsèquement formatrice parce que transformatrice des représentations, des croyances et des valeurs personnelles, mais aussi source majeure de socialisation par les terrains professionnels investis et à l'université, « avec » et " par » le groupe d'étudiants; autant d’opportunités de remise en cause et de construction de soi qu'offre le stage et que ne remplacent pas nécessairement d’autres modalités de formation, hybrides ou à distance, en autoformation ou par la validation d'acquis par exemple.

Une autre incidence pratique concerne le rôle des tuteurs de stage et des personnes participant au développement professionnel, qui n’a pas été détaillé ici. Quels "profils» de tuteurs et quels modes d’accompagnement participent le plus à ce développement ? Quelle durée de stage et quel rythme d'alternance, pour l'analyse des pratiques et la construction progressive de cette professionnalité, seraient les plus adaptés? Quel mode de collaboration entre tuteurs et enseignants mettre en place pour faire exister « le stage » en tant que modalité de formation à part entière et à égale reconnaissance des modalités dites " académiques " ? À l'heure où l'université est particulièrement interpelée sur sa capacité à prendre en compte la dimension sociale de ses formations, cette étude exploratoire d'une forme de pédagogie universitaire inspirée de la formation des adultes, nous laisse entrevoir tout son potentiel d'utilité professionnalisante pour l'université.

\section{Références bibliographiques}

Agulhon, C. (2007). La professionnalisation à l'université, une réponse à la demande sociale ? Recherche et formation (54), p. 11-28.

Altet, M. \& Bourdoncle, R. (2000). Formes et dispositifs de professionnalisation », dans Recherche et Formation (35).

Bardin, L. (2007). Lanalyse de contenu. Paris : PUF.

Beckers, J. (2007). Compétences et identité professionnelle. L'enseignement et autres métiers de l'interaction humaine. Bruxelles : De Boeck.

Bertrand, E. (2007). L’expérience formatrice à La Poste : vers une dialogique entre autoformation émancipatrice et coformation instrumentale.

Thèse inédite de doctorat en sciences de léducation, Université Paris 8.

Brémaud, L. \& Guillaumin, C. (2010). L'archipel de l'ingénierie de la formation : transformations, recompositions. Rennes : PUR.

Carré, P. (1992). L’autoformation dans la formation professionnelle. Paris : La Documentation Française.

Chi, M.T.H. (1997). Quantifying qualitative analysis of verbal data : a practical guide. The journal of the learning sciences, 6 (3), p. 271-315.

Charlier, B., Nizet J., et Van Dam, D. (2006). Voyage au pays de la formation des adultes : dynamiques identitaires et trajectoires sociales. Paris : L'Harmattan.

Clot, Y., \& Faïta, D. (2000). Genres et styles en analyse du travail. Concepts et méthodes, dans Travailler, (4), p. 7-42.

Crochard, M. (2007). Lalternance à l'université : rapport au savoir vs rapport au travail ? Éducation Permanente, (173), p. 83-94.

Day, C. (1999). Developing Teachers : The Challenges of Lifelong Learning. Londo : Falmer Press.

Dubar C. (2010). La socialisation. Construction des identités sociales et professionnelles. Paris : Armand Colin.

Eneau, J. (2011). Professionnaliser les métiers de la formation à l'université : de la "formation de formateurs" à la "formation à distance". Dans M. Moldoveanu (dir.), Les compétences des acteurs de léducation : perspectives internationales (195-212). Côte Saint-Luc (Québec) : Éditions Peisaj.

Eneau J., Bertrand E. \& Lameul G. (2011). Training the trainers at the University in time of crisis : implications of Transformative Learning for adult learning, educational programs and curriculum. Dans Proceedings of the 9th International Transformative Learning Conference in Europe 2011, Athènes (Grèce), 28-29 mai 2011, p. 596-603.

Eneau, J., Bertrand, E. \& Lameul, G. (2012), «Se former et se transformer : perspective critique et formation universitaire aux métiers de la formation. Revue internationale de pédagogie de l'enseignement supérieur, 28 (1). Téléacessible à l’adresse : http://ripes.revues.org/585.

Eneau, J. \& Develotte, C. (2012). Working online together to enhance learner autonomy. ReCALL, 24 (1), 2012, p. 3-19.

Eneau, J. \& Rey, D. (dir.). (2012). Université et métiers de la formation : quels enjeux, quelles spécificités ? Actes du Colloque du Réseau des Universités préparant aux Métiers de la Formation, Avignon, 12-13 mai 2011.

Epiphane, D. \& Giret, J.-F., (dir.). (2012). Introduction. Formation Emploi (117).

Fernagu-Oudet, S. (2007). Pour une alternance apprenante à l'université. Éducation Permanente, (173), p. 67-82.

Fessler, R. \& Christensen, J.C. (1992). The teacher career cycle : understanding and guiding the professional development of teachers. Boston : Allyn et Bacon.

Garet, M.S., Porter, A.C., Desimone, L., Birman, B.F. \& Suk Yoon, K. (2001). What makes professional development effective ? Results from a national sample of teachers. American Educational Research Journal, 38(4), p. 915-945.

Giret, J.-F. \& Issehnane, S. (2012). L'effet de la qualité des stages sur l'insertion professionnelle des diplômés de l'enseignement supérieur. Formation Emploi (« Enseignement supérieur : les défis de la professionnalisation, (117).

GREF (2012). Les trajectoires professionnelles des bénéficiaires du Programme régional des stages, Enquête IROISE, Contrat de Projets ÉtatRégion, Rennes, GREF Bretagne.

Herreros, G. (2012). La violence ordinaire dans les organisations. Plaidoyer pour des organisations réflexives. Toulouse : Erès. 
Jorro, A. (2009). Une posture de chercheur en lien avec la formation : la quête d'un éthos scientifique. Dans D. Bucheton (éd.), L'agir enseignant: des gestes professionnels ajustés (7-24). Toulouse : Octares.

Jorro, A. \& De Ketele, J.-M. (2011). La professionnalité émergente : quelle reconnaissance ? Bruxelles : De Boeck.

Kaddouri, M. (2002). La formation des adultes en entreprise : entre compétences et assignation identitaire. Les finalités de léducation. Éducation et francophonie, 30 (1), p. 158-171.

Kaddouri, M. (2006).Qu elques considérations transversales à propos de l'écriture sur sa pratique professionnelle. Dans F. Cros (dir.), Écrire sur sa pratique pour développer des compétences professionnelles (241-253). Paris : L'Harmattan.

Kaddouri, M. (2008). Quelques motifs identitaires d'engagement des professionnels dans des dispositifs de formation à la recherche », Dans C. Solar \& P. Hébrard (dir.), Professionnalisation et formation des adultes : une perspective universitaire France-Québec (157-195). Paris : L'Harmattan.

Kaddouri, M. (2010). Dynamiques identitaires et engagement en formation : lecture transversale d'une problématique de recherche. Note de synthèse présentée pour l'habilitation à diriger des recherches, Paris, Université Paris 13 Nord.

Kaddouri, M., Lespessailles, C., Maillebouis, M. \& Vasconcellos, M. (dir.). (2008). La question identitaire dans le travail et la formation. Paris : L'Harmattan.

Kerlinger, F. N. \& Lee, H. B. (2000). Foundations of Behavioral Research, (4e éd.). Holt, NY : Harcourt College Publishers.

Kuster, Y. \& Lameul, G. (2009). Un cadre d’analyse multidimensionnel de débats en ligne asynchrones. Dans C. Develotte, F., Mangenot, E. Nissen (coord.). Actes du colloque Épal «Échanger pour apprendre en ligne : conception, instrumentation, interactions, multimodalité. Université Stendhal - Grenoble3 (en ligne : http://w3.u-grenoble3.fr/epal/actes.html).

Lameul, G. (2006). Former des enseignants à distance ? Étude des effets de la médiatisation de la relation pédagogique sur la construction des postures professionnelles. Thèse inédite de doctorat en Sciences de l'Éducation, Paris : Université Paris Ouest La Défense.

Lameul, G., Eneau, J., Charlier, B. et al. (2011). Effets des dispositifs de formation hybrides sur le développement professionnel. Élaboration d'un cadre conceptuel et méthodologique dans la recherche Hy-Sup », dans Actes du Colloque «Questions de pédagogie dans l'Enseignement Supérieur », Angers, université d’Angers, p. 173-184.

Liaroutzos, O. (2012). L’alternance, un point de vue sur le travail. Éducation Permanente, (190), p. 81-90.

Maillard, F. (dir.). (2012). Former, certifier, insérer. Effets et paradoxes de l'injonction à la professionnalisation des diplômes. Rennes: PUR.

Martineau, S., Portelance, L. \& Presseau, A. (2009). La socialisation au travail comme indicateur de développement professionnel : analyse des approches basées sur la mesure. Questions Vives, 5(11). Téléacessible sur : http://questionsvives.revues.org/614.

Mayen, P. (2012). Questions d’apprentissage dans les formations par alternance. Éducation Permanente, (193), 2 p. 49-69.

Mezirow, J. (2001). Penser son expérience. Une voie vers l'autoformation. Lyon : Chronique Sociale.

Pratt, D. (and ass.). (1998). Five Perspectives on Teaching in Adult and Higher Education. Malabar (FL), :Kreiger.

Simard, D. (2010). La culture professionnelle des enseignants : entre savoirs, recherches et pratiques. Recherches en éducation, Hors Série $\mathrm{n}^{\circ} 2$.

Wittorski, R. (2007). Professionnalisation et développement professionnel. Paris : L'Harmattan.

Wittorski, R. (2008). La professionnalisation. Note de synthèse. Savoirs, 17(2), p. 9-36.

Wittorski, R. (dir.). (2010). Regards croisés sur la professionnalisation et ses objets. Les dossiers des Sciences de l'Éducation, (24).

Wittorski, R., (dir.). (2012). Formation et professionnalisation», dans Éducation Permanente, (188).

Wittorski, R. (2012). La professionnalisation de l'offre de formation universitaire : quelques spécificités. Revue internationale de pédagogie de lenseignement supérieur, 28 (1) Téléacessible sur : http://ripes.revues.org/580.

Zaouani-Denoux, S. (2007). Formation et construction de soi : de l’alternance à l'“altérance”. Éducation Permanente, (172), p. 73-80. 